\title{
Research on Key Techniques of Command and Dispatch in Hybrid Voice Communication
}

\author{
Linying Geng, Dengwei Chen, Xinhai Guo, Guangchao Zhao, Rui Sun \\ China Luoyang Experiment Center of Electronic Equipment, 471023, China
}

Keywords: mobile communication; wire dispatch; signaling detection

\begin{abstract}
This paper is based on a platform of business convergence, this paper designs and improves the access of the wireless dispatch communication network and other mobile communication devices (such as walkie talkie, etc.) based on the wired dispatch communication network to form a command and dispatch system of hybrid voice communication. The system adopts signaling detection technology, which is more reliable, stable and practical. The function of command and dispatch appears more flexible and reliable through the trunk access PSTN telephone network. At present, the program has been used in various tasks of China Luoyang Experiment Center of Electronic Equipment to achieve smooth command and dispatch.
\end{abstract}

\section{Introduction}

The companies of commanding the front-line work are facing the coexistence of digital cluster and analog cluster equipment, the relative lagging of technical research, and practical difficulties that completely updating equipment is expensive. How to combine the existing equipment to reform the equipment, optimize the technical scheme, make the communication system of command and dispatch with requirements has become the the only way of current development. A hybrid communication system of command and dispatch is proposed, which has the features of full function, high reliability, strong anti-interference ability and good call quality compared with the domestic research [1]. The system has been widely used in a variety of comprehensive testing tasks, and has been proved to be promising.

\section{General System Scheme}

The research of this paper is based on BY-AVSP-TS004-1 audio fusion dispatching device (hereinafter referred to as "audio fusion device"). The audio fusion device is mainly composed of universal controller chassis and various boards. The universal controller chassis is the container of various boards. It supplies power for boards, provides data exchange among boards, and provides the corresponding external interface for each board. However, the audio fusion device only equips with two function of that program-controlled telephone dials four-wire analog dispatching, and program-controlled telephone calls cluster handset. And it doesn't equip with the function of three party service. It cannot achieve those functions of dispatch terminal, cluster base station, interconnection of Program - controlled exchange and hybrid audio conference.

In the current use of audio system, the mode of the dispatching system and telephone network is full duplex communication system, in which there are difficulties on the intercommunication. The public telephone network is connected into the fusion platform through E1 digital trunk; the wireless dispatching system of Motorola (Hereinafter referred to as "wireless dispatching system") has not external interface, when external system voice inputting and internal system voice outputting by the means of system vehicle-borne platform, there are some problems as follows: timely audio controlling of half duplex, duplex system according to the need of the communication, if there is voice input detected, the audio fusion system will automatically trigger the PTT function to seize the channel, then release the channel after the voice input is finished to ensure the automatic building of the full-duplex and half-duplex communication system. This is also a problem of intercommunication between half-duplex and full-duplex systems. Since there are many users in the external system and there is no PTT button, it is not certain when someone speaks. Therefore, the 
PTT of the vehicle-borne platform can only be pressed, so that other terminal devices in the wireless dispatching system can only receive voice and input voice.

In order to solve the above problems, the following solution is proposed: the half-duplex system is connected through PTT interface, while the PTT signal is converted into SIP signaling message, and the unified control of soft-switching is accepted. In this way, the audio fusion system can realize timely voice control of the half-duplex system according to the communication requirement. Soft switch can activate the voice channel of the half-duplex system by signaling when the scheduling task is executed to ensure automatic call setup between the full duplex mode communication system and the half-duplex system.

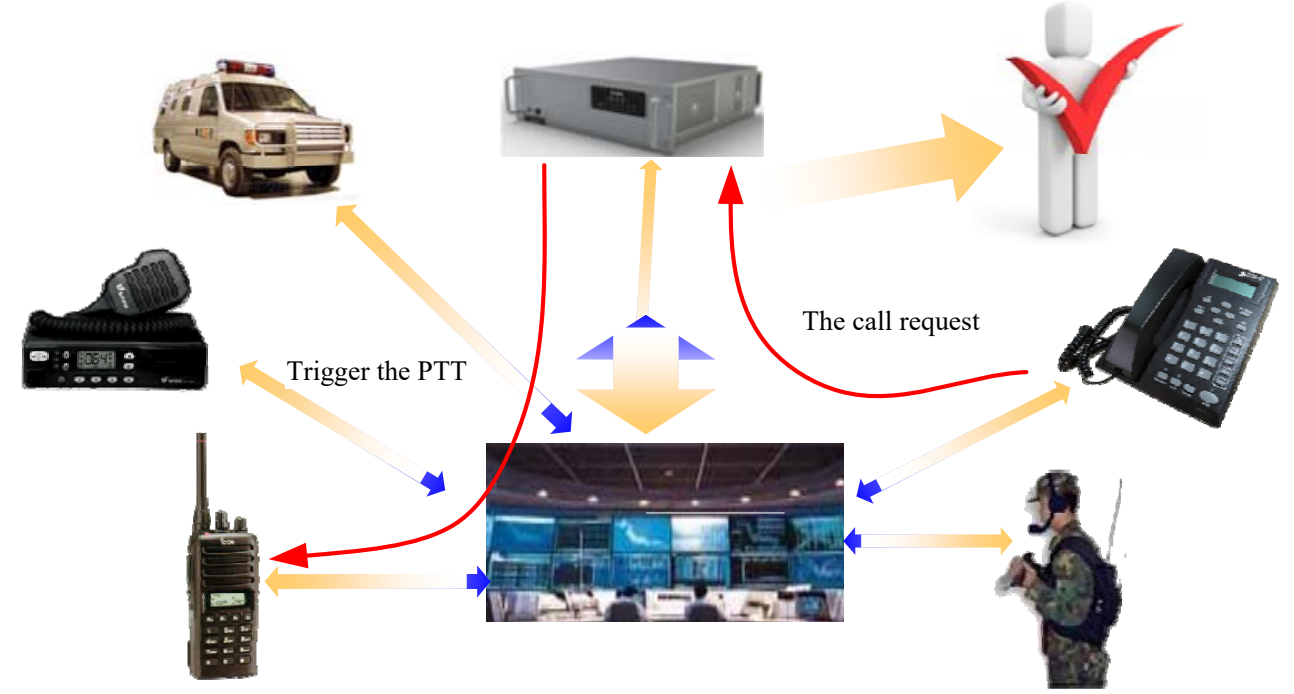

Figure1 Schematic diagram shows the call connection between of the user of telephone network and the users of wireless security dispatch.

Take figure 1, that the user of telephone network calls the user of wireless security dispatch as the example, the audio fusion device can convert the call request of the telephone network users starting through the 7th signaling into the corresponding PTT signal to trigger the cluster call building of the wireless security dispatch and then achieve the cluster call channel building between users of the telephone network and users of the wireless security dispatch. In the audio fusion device, the call request of users of the telephone network through the based on the $7^{\text {th }}$ signaling is converted into the standard SIP signaling in the first, then start exchanging the addressing information into the corresponding access module, and that the corresponding access module identifies the corresponding SIP signaling trigger corresponding PTT event, thus a voice channel is built.

\section{System Implementation}

\section{The base station of wireless dispatching system and the design of audio fusion device interface}

The external of system's base station and vehicle-borne platform only have audio input and output and PTT input interface, and the fusion device does not support two interfaces. The audio input and output interface type of wireless security dispatch system equipment is DB9 serial port, in order that the fusion device connects to the base station, we reform the serial port to achieve the mutual connection among systems. We produced the DB9 interface audio cable, adopting 16-pin interface, the first pin audio output, the second pin audio input, the $3^{\text {rd }}, 7^{\text {th }}$ pin connection PTT function, to achieve the interconnection between the two systems.

The original base station is input into the ECU board and short DB9 head of the third, fourth pin through the DB9 interface, the fourth pin and fifth pin.PTT input terminal of ECU board, the seventh line (white line) joining into DB9 line are connected to the base station terminal, the PTT output terminal of ECU board is connected to the second line (orange line) of DB9 line. All the 
ground wires are connected to the first line (red wire) of DB9 line. Audio fusion equipment provides voice input and output, the use of PTT input and output signals connects to the wireless security dispatching cluster base station through the DB9 line. Pin arrangement and definition of base station rear interface shall be shown in Table 1 and Table 2:

Table 1 The pins list of base station rear interface

\begin{tabular}{|l|l|l|l|l|l|l|l|l|l|}
\hline 20 & 2 & 4 & 6 & 8 & 10 & 12 & 14 & 16 SPKR + & 18 \\
\hline 19 & 1SPKR- & 3 & 5 & 7 & 9 & 11 & 13 & 15 & 17 \\
\hline
\end{tabular}

Table 2 The definition list of BS rear interface pins

\begin{tabular}{|l|l|l|}
\hline Pin & Definition & Type \\
\hline 1 & SPKR- & Analog signal output \\
\hline 2 & EXT. MIC Audio & Analog input \\
\hline 3 & GPIO 1 (5V) & Digital input \\
\hline 4 & GPIO 2 (13v) & Value output \\
\hline 5 & Flat TX Audio & Analog input \\
\hline 6 & GPIO 3 (5V) & Digital input \\
\hline 7 & GND & Ground \\
\hline 8 & GP3 GPIO 4 (5V) & Digital input /output \\
\hline 9 & GP4 GPIO 5 (5V) & Digital input \\
\hline 10 & & \\
\hline 11 & Ignition Sense & Digital input \\
\hline 12 & Receive Audio & Analog output \\
\hline 13 & GP5 GPIO 7 (5V) & Digital input /output \\
\hline 14 & Power Output & Analog output \\
\hline 15 & GP6 GPIO 8 (5V) & Analog input/output \\
\hline 16 & RSSI & Analog output \\
\hline 17 & SPKR+ & Analog output \\
\hline 18 & BOS+ USB & Digital input /output \\
\hline 19 & NC & Digital input \\
\hline 20 & NC & Blank \\
\hline
\end{tabular}

The actual use of 1-16 pin, in which the seventh pin is the ground line, the forth pin is audio output, the third pin and the fifth pin is audio input, the first pin and $16^{\text {th }}$ pin audio output without white noise. The fourth pin as audio input in the wireless security dispatching system is connected to the encryptor, when designing, the input routine of the audio is changed to remove the impact of white noise on the system, for the first pin and $16^{\text {th }}$ pinas audio connected to the audio fusion equipment.

Achieve connecting to audio fusion equipment through the base station:

Wireless dispatch system Base station is actually composed of two vehicle-borne platforms, in which one is the receiving device, mainly for voice reception, one is the sending device, mainly for voice transmission. Equipment manufacturers take use of the vehicle-borne platform to provide the expansion interface, to achieve the interaction between the vehicle-borne platforms [2]. Figure 2 shows an internal schematic diagram of the dispatching system base station of the wireless security.

Base station equipment takes full advantage of the expansion interface of the vehicle-borne platforms to achieve the trunk function of radio waves through controlling circuit board. When the PTT button of the terminal equipment is pressed, the receiving vehicle-borne platforms receives the carrier signal and the carrier detection signal line of the expansion interface generates a high level. After detecting the level, the control circuit board sends the PTT signal to the transmitting vehicle-borne platforms, and the received voice is sent out. 


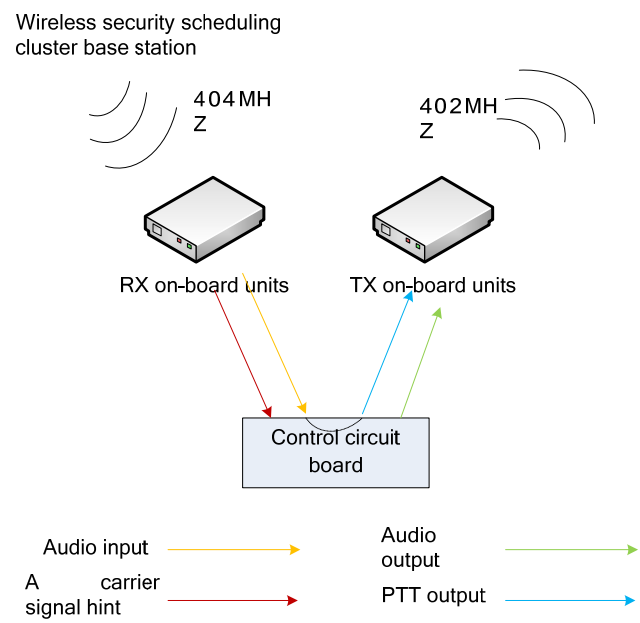

Figure 2 The internal schematic diagram of wireless dispatching system base station

$\mathrm{RX}$ vehicle-borne platforms is inputted into the audio fusion device in the internal of base station, and then mix with other voice of other systems (the three-party call logic of MGU2421), after that, which is outputted into TX vehicle-borne platforms to reach the purpose of simultaneous speech of users among the terminal equipment and other voice system. Figure 3 shows a schematic diagram of a wireless security dispatch system docking with an audio fusion device through a base station.

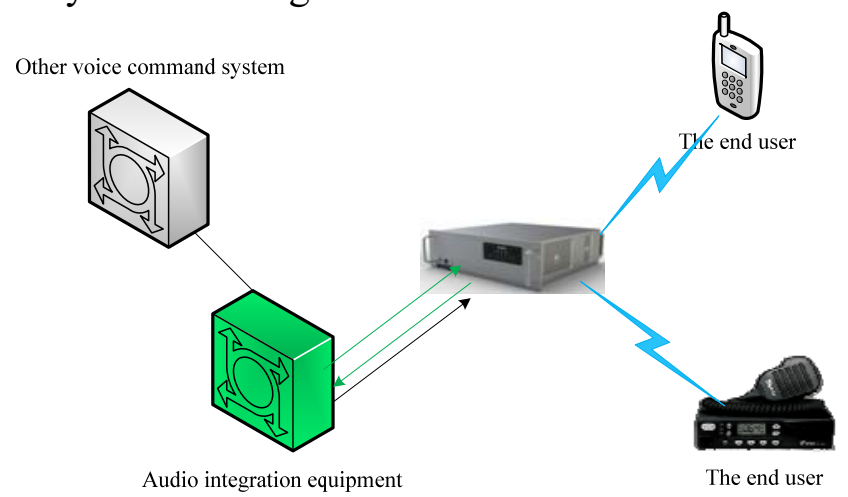

Figure 3 The diagram II that wireless security dispatching system docking with audio fusion equipment

\section{Half-duplex and full-duplex call control}

Wireless dispatching cluster system does not support the trunk function, only support the half-duplex communication mode controlled by the PTT, for the external, the base station and vehicle-borne platforms only have audio input and output and PTT input interface. Audio fusion devices can only access the wireless security dispatching cluster via the audio and PTT lines of the vehicle-borne platforms, as shown in Figure 4:

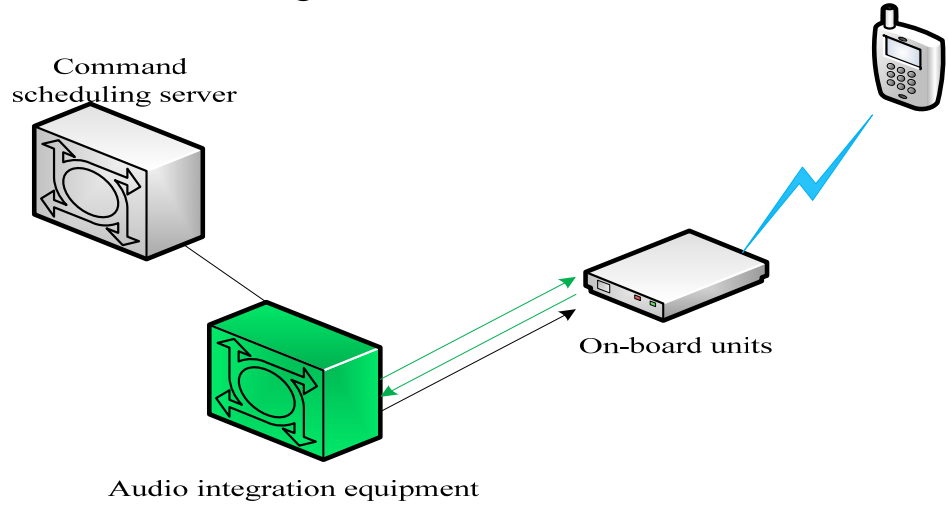

Figure 4 The connection relation of wireless security dispatching cluster

If the voice of the dispatching side is sent to the wireless side, you shall press the PTT button, and at the same time the wireless side cannot utter voice. As the system cannot detect when 
someone speaks on the dispatching side, so either we keep the PTT of the vehicle-borne platforms pressing or keep it releasing all the time, which can only achieve single-party talk. As Figure2.

Referring to the internal structure of wireless dispatching base station, you can propose solutions to the above problems. As shown in Figure 5:

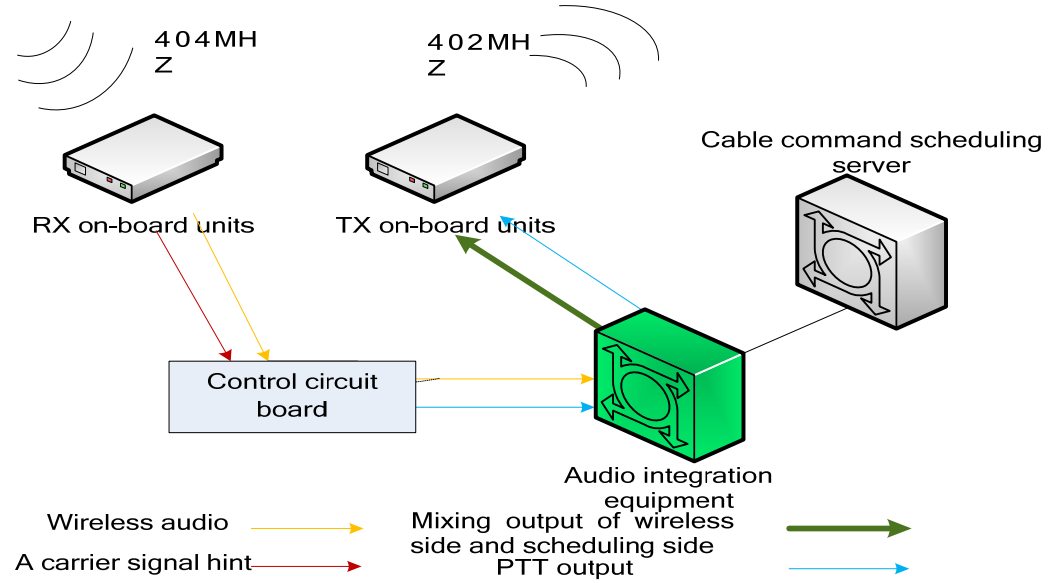

Figure5 Schematic diagram of the proposed solution

When the wireless dispatching system cluster is in the conference, the voice of RX vehicle-borne platform in base station is inputted into the audio fusion equipment, and mix with the voice of the dispatching side, after that, which is outputted into the TX vehicle-borne platform, to reach the purpose of simultaneous speech of users among the terminal equipment and the dispatching side.

\section{Three-party call flow}

For the wireless security dispatching cluster, the users in the cluster can make internal calls through the handheld terminal. While the external system (such as participant 1 in Figure 6) communicating with and the wireless security dispatching cluster (participant 2), the users in the wireless dispatching cluster can hear the voice of other users in the internal system, and also can hear the voice if the external system, which forms a multi-party call scene.

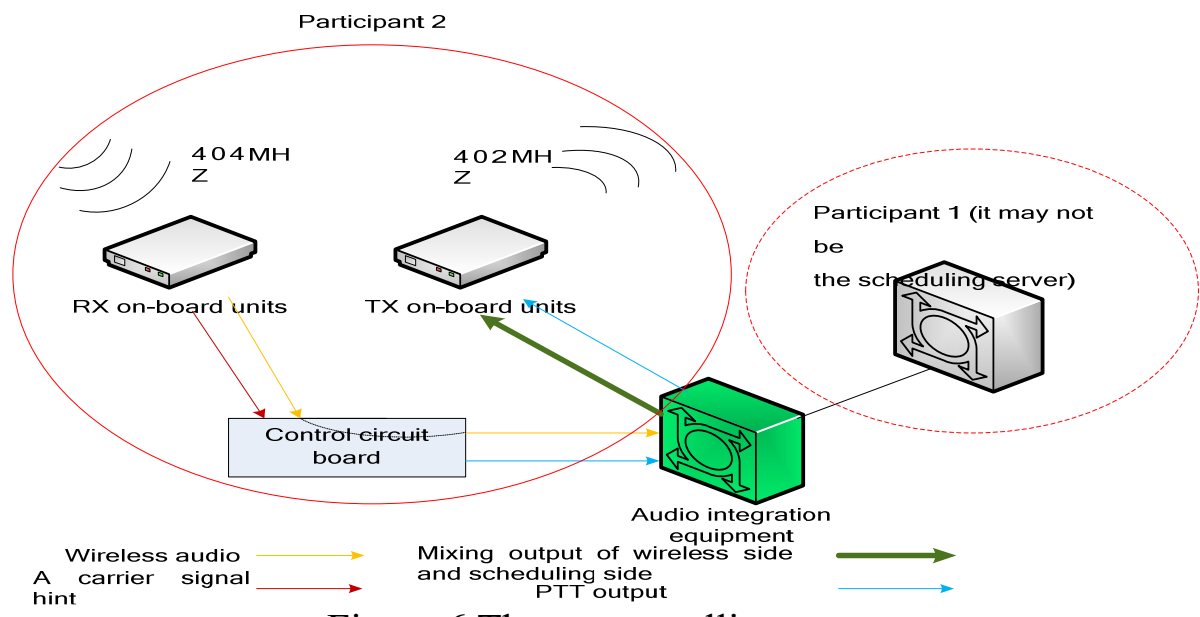

Figure 6 Three-way calling scene

\section{Conclusion}

The communication system of hybrid command and dispatch proposed in this paper is connected to the wireless dispatching communication system on the communication network with the audio fusion equipment as the core, and access PSTN (public switched telephone network) through trunks[3].The system uses the signaling detection, processing and control technology in the wired / wireless interface of the system to improve the reliability, stability and practicability of the system. At present, the system has been applied to a variety of integrated test tasks, through performance 
testing, functional testing, communication test, the performance indicators and control functions, all that can meet the demand, and has the characteristics of full-featured, high reliability, anti-interference ability, good call quality[1][4], compared with similar domestic products, the system has a better development prospect[5].

\section{Reference}

[1] Hu Haibo \& Wang Jiqing \& Lu Kunsheng \& Qin Zheng. Implementation of a Wired / Wireless Mixed Command and Dispatch Communication System. Electronic Quality. Issue 5. 2004

[2] Zhang Weiwei. CTCS3 level control system simulation test platform - on-board equipment simulation subsystem. Beijing Jiaotong University. 2006

[3] Hu Haibo.Design and Implementation of a Mixed Command / Dispatch Communication System [D]. Huazhong University of Science and Technology. 2005.

[4] Yuan Long. TruStar: a new generation of digital trunking communication star. Communication World B. Issue 7. 2008

[5] Ye Qiping. SC knowledge management and performance evaluation of the exploration. Shanghai Jiaotong University. 2001 